\title{
Proteinuria Level and Associated Changes in Glomerular Podocytes and Renal Tubular Epithelium
}

\author{
Maha M Arafah* \\ Department of Pathology, College of Medicine, King Saud University, Kingdom of Saudi Arabia
}

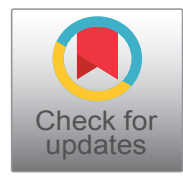

*Corresponding author: Maha Arafah, MBBS, KSUFP, Associate Pathologist, Histopathology, Cytology and Electron Microscopy Unit, Department of Pathology, College of Medicine, King Saud University, PO Box: 7805, Riyadh, 11472, Kingdom of Saudi Arabia, Tel: +966114670360 , Fax: +966114672462

\begin{abstract}
The correlation between glomerular podocytes and renal tubular cells involved in handling of leaked proteins and the level of proteinuria was evaluated. Retrospective 22 cases of clinical proteinuria in patients with various glomerulopathies were retrieved and analyzed. Glomerulopathies in the concerned patients were pathologically diagnosed through light and electron microscopic examination of the submitted renal biopsies. Three cases with protein levels in urine within the acceptable normal range were additionally analyzed as controls. Electron microscopic examination of the glomerular podocytes and immunofluorescence of the renal tubular epithelium in the relevant cases constituted the base for the present study. Among the studied cases, it was found that the greater the number of glomerular podocytes with reabsorbed intracytoplasmic proteins and the higher score of tubular protein immunofluorescence, the lower the level of proteinuria. Comparatively, cases with fewer number of podocytes with reabsorbed proteins and lower score of tubular protein immunofluorescence had higher levels of proteinuria.

The present study aims to pay the attention to the correlation between morphologically recognizable glomerular podocytes and renal tubular epithelium with reabsorbed proteins and the level of proteinuria in patients with various glomerulopathies. The current study may serve as a base for the future research work concerned with the structural changes of glomerular podocytes and renal tubular epithelium as a compensative mechanism in cases of proteinuria.
\end{abstract}

\section{Keywords}

Podocytes, Tubular epithelium, Reabsorbed proteins, Proteinuria

\section{Introduction}

Podocytes (glomerular visceral epithelial cells) are highly specialized cells constituting a crucial component of the three-layered glomerular filtration barrier of the kidney [1,2]. Failure of podocyte function is involved in the progression of chronic glomerular disease [3]. Renal tubular cells are actively engaged in reabsorption of proteins that may leak into urine due to disruption of the glomerular filtration barrier [1-7].

Proteinuria is the condition characterized by the presence of greater than normal amounts of proteins in the urine [4-6,8-11]. There are 3 main causes of proteinuria, namely; glomerular diseases, increased quantity of proteins in serum (overflow proteinuria), and low reabsorption of the renal proximal tubules $[7,12,13]$. In case of glomerular diseases, the glomerular filtration barrier is damaged permitting proteins such as albumin to leak from the blood into the urine. Proteinuria can be classified on the basis of protein amount (nephrotic or non-nephrotic), on the type of protein (albuminuria or low molecular weight proteinuria), or on the underlying pathological changes (glomerular vs. non-glomerular) [14-16]. Low protein reabsorption of the renal proximal tubules is one of the three main causes of proteinuria [7,12]. Renal tubular dysfunction is accordingly classified as a non-glomerular contributing factor in the development of proteinuria. The aim of the present retrospective study is to evaluate the correlation between the glomerular podocytes and renal tubular epithelium containing reabsorbed proteins, as recognizable by transmission electron

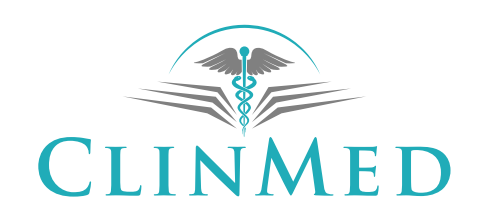

INTERNATIONAL LIBRARY
Citation: Arafah MM (2019) Proteinuria Level and Associated Changes in Glomerular Podocytes and Renal Tubular Epithelium. Int J Pathol Clin Res 5:088. doi.org/10.23937/2469-5807/1510088

Accepted: April 27, 2019: Published: April 29, 2019

Copyright: (C) 2019 Arafah MM. This is an open-access article distributed under the terms of the Creative Commons Attribution License, which permits unrestricted use, distribution, and reproduction in any medium, provided the original author and source are credited. 
microscopy and immunofluorescence, and the level of proteinuria in patients with various glomerulopathies.

\section{Materials and Methods}

\section{Patients}

The study was conducted in Anatomic Pathology Section, Department of Pathology and Laboratory Medicine, College of Medicine, King Saud University, over a period extending from January, 2013 to December, 2015. Twenty-two patients with clinical proteinuria were enrolled in the study with the involvement of additional three patients with protein levels in urine within the acceptable normal range as controls. At the time of sample collection, none of the concerned patients received any treatment that may influence the results of urine samples analysis.

\section{Histopathology}

Renal biopsies obtained from all patients were processed for light microscopic examination and all staining protocols, including the routine and immunofluorescence staining, were carried out.

\section{Electron microscopy}

Transmission electron microscopic examination of the renal biopsies was performed to examine the glomerular podocytes at the ultrastructural level. Briefly, renal tissues were primarily fixed in $2.5 \%$ buffered glutaraldehyde (phosohate buffer, $\mathrm{pH} 7.2$ ) and post-fixed in $1 \%$ osmium tetroxide (OsO4). Tissues were then dehydrated in ascending series of ethyl alcohol and subsequently embedded in epoxy resin (Epon: Araldite mixture). Semi-thin tissue sections ( $0.5 \mu \mathrm{m}$ thickness) were made and accordingly ultra-thin sections $(70-85 \mathrm{~nm}$ thickness) were prepared and double stained with uranyl acetate and lead citrate. Ultra-thin tissue sections were examined and photographed under a transmission electron microscope (TEM) (JEM-1400 TEM, JEOL Co., Tokyo, Japan) operating at $100 \mathrm{kV}$.

\section{Counting of glomerular podocytes}

The appropriate methods, including Weibel and Gomez point counting method, described in previous studies $[9,17,18]$ to measure the proportion of glomerular cell types were employed. Accordingly, the glomerular podocytes were counted and the average number of podocytes/glomerulus in each of the examined cases was calculated. The average number of podocytes containing reabsorbed intracytoplasmic proteins/glomerulus was also estimated.

\section{Immunofluorescence}

Reabsorbed proteins (albumin) in renal tubular cells were the target immunofluorescence (IF) staining. Serial frozen sections were prepared from the unfixed renal tissues and treated with a fluorescein-labelled antibody specific for albumin. The intensity of IF staining in renal tubular cells was expressed semi-quantitatively as: negative, no reabsorption proteins; mild, less than $25 \%$ of the tubules show reabsorption proteins; moderate, $26 \%$ to $50 \%$ of the tubules show reabsorption proteins; and severe, more than $50 \%$ of the tubules show reabsorption proteins. The corresponding reabsorbed albumin scores as observed by light microscopy in renal tubular cells were: 0 , no significant hyaline cytoplasmic reabsorption droplet change; $1+$, Minimal hyaline reabsorption droplet change; $2+$, Mild hyaline reabsorption droplet change; $3+$, Moderate hyaline reabsorption droplet change.

\section{Statistical analysis}

Data on counting of glomerular podocytes with reabsorbed proteins, immunofluorescence scoring of reabsorbed proteins and albumin scores in the renal tubular cells, and proteinuria levels were recorded and presented as mean and standard deviation. Statistical analysis of the data was done using Wilcoxon Mann-Whitney $U$ test $(p$-value $=0.035)$.

\section{Results}

Immunofluorescence (IF), electron microscopic findings, and the pathological diagnosis for each of the studied 25 cases was summarized in (Table $1 \mathrm{~A}$ and Table 1B). Because of the existing variations of disease etiology, the studied cases were divided into 2 groups; the first group (Table 1A) encompassed those cases with immunocomplex deposition-related glomerulonephritis, and the second one involved those with non-immunocomplex-related glomerulopathies.

In (Table 2), demonstrate the level of proteinuria, average number of podocytes/glomerulus, and average number of podocytes with reabsorbed intracytoplasmic proteins/glomerulus in 25 patients with various glomerulopathies. The average number of podocytes/glomerulus was variable among the studied disease entities and ranged from 20-26. The average number of podocytes with reabsorbed intracytoplasmic proteins/glomerulus ranged from 0-5. The highest average number of podocytes with distinct reabsorbed proteins/glomerulus (3-5 cells/glomerulus) were found in cases with low proteinuria levels (0.49-2.32 gm/day). Cases with the average number of only one podocyte with reabsorbed proteins/glomerulus revealed the highest level of proteinuria ranging from $4+$ to $28.97 \mathrm{gm} /$ day.

Contents of (Table 3 ) clearly indicated that cases in high number group (three-five podocytes with reabsorbed proteins/glomerulus) manifested the lowest level of proteinuria, while the highest level of proteinuria was encountered in cases of low number group (one podocyte/glomerulus).

Levels of proteinuria, tubular immunofluorescence and albumin scores of reabsorbed proteins in the renal tubular epithelium as well as tubular cell injury in each case were shown in (Table 4). 
Table 1A: Immunoflourescence, electron microscopic findings, and pathological diagnosis in 25 patients with various glomerulopathies.

A. Immunocomplex deposition-related glomerulonephritis.

\begin{tabular}{|c|c|c|c|c|c|c|}
\hline & \multirow[t]{2}{*}{$\begin{array}{l}\text { Case } \\
\#\end{array}$} & \multirow[t]{2}{*}{ Age } & \multirow[t]{2}{*}{$\begin{array}{l}\text { Sex } \\
\text { M:male } \\
\text { F:female }\end{array}$} & $\begin{array}{l}\text { Immunoflourescence } \\
\text { Immunoglobulin and } \\
\text { complement }\end{array}$ & $\begin{array}{l}\text { Electron } \\
\text { Microscopic } \\
\text { Findings }\end{array}$ & $\begin{array}{l}\text { Pathological } \\
\text { Diagnosis }\end{array}$ \\
\hline & & & & $\begin{array}{l}\text { Effacement of the } \\
\text { epithelial cell foot } \\
\text { processes }\end{array}$ & $\begin{array}{l}\text { Electron dense } \\
\text { immune deposits } \\
\text { and other findings }\end{array}$ & \\
\hline 1 & 33 & $\mathrm{~F}$ & $\begin{array}{l}\text { Diffuse mesangial and focal } \\
\text { capillary positivity lgG }(2+) \text { and IgM } \\
(1+) \text {. } \\
\text { finely granular diffuse capillary and } \\
\text { focal mesangial positivity for C3 } \\
(2+) \text {, C1q }(2+) \text {, Kappa }(1+) \text { and } \\
\text { lambda }(2+)\end{array}$ & Diffuse & $\begin{array}{l}\text { Subepithelial, } \\
\text { intramembranous, } \\
\text { subendothelial and } \\
\text { mesangial deposits }\end{array}$ & Lupus nephritis \\
\hline 3 & 50 & M & $\begin{array}{l}\text { Focal mesangial and capillary } \\
\text { deposition of IgA }(1+) \& \text { Lambda } \\
(1+) \text { and focal mesangial deposition } \\
\text { of C3 }(1+) \text { in the glomeruli. }\end{array}$ & Extensive patchy & $\begin{array}{l}\text { Electron dense } \\
\text { deposits in the } \\
\text { mesangium and } \\
\text { Focal double } \\
\text { contouring }\end{array}$ & $\begin{array}{l}\text { Consistent with } \lg A \\
\text { nephropathy }\end{array}$ \\
\hline 4 & 34 & $\mathrm{~F}$ & $\begin{array}{l}\text { Diffuse linear capillary positivity } \\
\text { for IgA }(1+) \text {, IgG }(3+) \text {, C3 }(3+) \text {, } \\
\text { C1q }(3+), \text { kappa }(2+) \text { and lambda } \\
(2+) \text {. There is focal capillary and } \\
\text { mesangial positivity for IgM }\end{array}$ & Extensive patchy & $\begin{array}{l}\text { Subendothelial } \\
\text { electron dense } \\
\text { deposits }\end{array}$ & $\begin{array}{l}\text { Immune complex } \\
\text { mediated disease with } \\
\text { membrano-proliferative } \\
\text { pattern of injury }\end{array}$ \\
\hline 5 & 17 & $\mathrm{~F}$ & $\begin{array}{l}\lg A \text { and IgM: moderate }(3+), \operatorname{lgG} \text {, } \\
\text { C1q and C } 3 \text { : severe }(4+), \text { Kappa } \\
\text { and Lambda: severe }(4+) \text { diffuse } \\
\text { mesangial and diffuse capillary } \\
\text { positivity }\end{array}$ & Extensive patchy & $\begin{array}{l}\text { Mesangial, } \\
\text { paramesangial and } \\
\text { subendothelial areas }\end{array}$ & Lupus nephritis \\
\hline 8 & 67 & M & $\begin{array}{l}\text { IgA: Mild }(2+) \text { extensive mesangial } \\
\text { positivity }\end{array}$ & Diffuse & $\begin{array}{l}\text { Mesangium and } \\
\text { paramesangium }\end{array}$ & IgA nephropathy \\
\hline 9 & 25 & $\mathrm{~F}$ & $\begin{array}{l}\lg A, \lg G \text { and } \operatorname{lgM}: 2+\text { diffuse } \\
\text { mesangial positivity. } \\
\text { C3 and C1q: } 3+\text { diffuse granular } \\
\text { capillary and mesangial }\end{array}$ & Extensive diffuse & $\begin{array}{l}\text { Subendothelial, sub } \\
\text { epithelial, mesangial } \\
\text { and paramesangia }\end{array}$ & Lupus nephritis. \\
\hline 10 & 20 & $\mathrm{~F}$ & $\begin{array}{l}\text { IgA and IgG: Negative. IgM: } 1+ \\
\text { focal mesangial and capillary } \\
\text { positivity. C3: } 2+\text { diffuse capillary } \\
\text { and focal mesangial positivity. } \\
\text { Focal vascular positivity }\end{array}$ & Diffuse & $\begin{array}{l}\text { Glomerular } \\
\text { basement } \\
\text { membrane, } \\
\text { mesangial and } \\
\text { paramesangial } \\
\text { areas and ribbons } \\
\text { of marked electron } \\
\text { dense deposits }\end{array}$ & $\begin{array}{l}\text { Crescentic } \\
\text { glomerulonephritis } \\
\text { secondary to Dense } \\
\text { Deposit Disease (C3 } \\
\text { Glomerulopathy). }\end{array}$ \\
\hline 12 & 48 & M & $\begin{array}{l}\text { IgA and IgM: Negative. IgG: } 2+ \\
\text { diffuse coarse granular capillary } \\
\text { positivity. C3 and C1q: Negative. } \\
\text { Kappa: } 2+\text { diffuse capillary positivity } \\
\text { Lambda: } 2+\text { focal capillary positivity }\end{array}$ & Diffuse & Intramembranous & $\begin{array}{l}\text { Membranous } \\
\text { glomerulonephritis }\end{array}$ \\
\hline 13 & 48 & M & $\begin{array}{l}\text { IgA, IgM, C3: Moderate extensive } \\
\text { mesangial positivity } \\
\text { IgG: Negative. Kappa: Mild (2+) } \\
\text { focal mesangial positivity. } \\
\text { Lambda: Moderate }(3+) \text { extensive } \\
\text { mesangial positivity }\end{array}$ & Extensive diffuse & $\begin{array}{l}\text { Mesangial and } \\
\text { paramesangial } \\
\text { areas }\end{array}$ & IgA nephropathy. \\
\hline
\end{tabular}




\begin{tabular}{|c|c|c|c|c|c|c|}
\hline 14 & 25 & $\mathrm{~F}$ & $\begin{array}{l}\text { IgM: Moderate }(3+) \text { extensive } \\
\text { mesangial positivity } \\
\text { C3: Severe }(4+) \text { extensive } \\
\text { mesangial positivity. } \\
\text { IgA and IgG negative }\end{array}$ & Focal & $\begin{array}{l}\text { Mesangial and } \\
\text { paramesangial } \\
\text { areas and sub- } \\
\text { endothelial deposits } \\
\text { with double contour } \\
\text { formation }\end{array}$ & $\begin{array}{l}\text { C3 glomerulonephritis } \\
\text { with a } \\
\text { mesangioproliferative } \\
\text { pattern. }\end{array}$ \\
\hline 16 & 16 & M & $\begin{array}{l}\text { IgM: 2+ diffuse mesangial } \\
\text { IgA, IgG, C3 and C1q: Negative. } \\
\text { Kappa and Lambda: Negative }\end{array}$ & Diffuse & $\begin{array}{l}\text { Mesangium and } \\
\text { paramesangium } \\
\text { with Microvillous } \\
\text { degeneration }\end{array}$ & IgM nephropathy \\
\hline 17 & 6 & M & $\begin{array}{l}\text { IgA, IgG, C3 and C1q: Negative. } \\
\text { IgM: 2+ extensive mesangial }\end{array}$ & Focal & $\begin{array}{l}\text { Mesangial and } \\
\text { paramesangial } \\
\text { areas }\end{array}$ & IgM nephropathy \\
\hline 18 & 36 & $\mathrm{~F}$ & $\begin{array}{l}\text { IgA: } 1+\text { diffuse capillary } \\
\text { IgG: } 3+\text { diffuse granular capillary } \\
\text { IgM: } 1+\text { focal capillary } \\
\text { C3: } 1+\text { diffuse capillary and Focal } \\
\text { arteriolar positivity } \\
\text { C1q: } 1+\text { diffuse capillary } \\
\text { Kappa and Lambda: } 2+\text { diffuse } \\
\text { capillary }\end{array}$ & Diffuse & $\begin{array}{l}\text { Subepithelial and } \\
\text { intramembranous } \\
\text { electron }\end{array}$ & $\begin{array}{l}\text { Membranous } \\
\text { nephropathy }\end{array}$ \\
\hline 19 & 16 & M & $\begin{array}{l}\text { IgA, IgG, IgM, C3 and C1q: } \\
\text { moderate }(3+) \text { diffuse mesangial } \\
\text { and focal capillary } \\
\text { Kappa and Lambda: moderate }(3+) \\
\text { diffuse mesangial }\end{array}$ & Diffuse & $\begin{array}{l}\text { Subendothelial, } \\
\text { mesangial and } \\
\text { paramesangial } \\
\text { areas }\end{array}$ & Lupus nephritis \\
\hline 20 & 50 & $\mathrm{~F}$ & $\begin{array}{l}\text { IgA and IGM: Trivial }(1+) \text { focal } \\
\text { mesangial } \\
\text { IgG, C3 and C1q: Mild }(2+) \text { focal } \\
\text { mesangial }\end{array}$ & Little focal & $\begin{array}{l}\text { Large subendothelial } \\
\text { deposit and a few } \\
\text { mesangium and } \\
\text { paramesangium }\end{array}$ & Lupus nephritis. \\
\hline 21 & 2 & $\mathrm{~F}$ & $\begin{array}{l}\text { IgA and IgG: Trivial (+/-) focal } \\
\text { mesangial positivity } \\
\text { IgM: Moderate }(3+) \text { diffuse } \\
\text { mesangial positivity in all glomeruli. } \\
\text { C3 and C1q: Negative. } \\
\text { Kappa and Lambda: Trivial (1+) } \\
\text { focal mesangial positivity. }\end{array}$ & Diffuse & $\begin{array}{l}\text { Mesangial and } \\
\text { paramesangial } \\
\text { areas }\end{array}$ & IgM Nephropathy \\
\hline 22 & 49 & $\mathrm{~F}$ & $\begin{array}{l}\operatorname{Ig} A: 2+ \\
\lg \text {, IgM, C3 and C1q: Negative }\end{array}$ & Extensive diffuse & $\begin{array}{l}\text { Mesangial and } \\
\text { paramesangial } \\
\text { areas. }\end{array}$ & IgA nephropathy \\
\hline 23 & 46 & $M$ & $\begin{array}{l}\text { IgA : 3+ } \\
\text { IgG, IgM, C3 and C1q: Negative } \\
\text { Kappa: } 1+ \\
\text { Lambda: } 2+\end{array}$ & Focal & $\begin{array}{l}\text { Mesangial and } \\
\text { paramesangial } \\
\text { areas. }\end{array}$ & IgA nephropathy \\
\hline 24 & 30 & $\mathrm{~F}$ & $\begin{array}{l}\text { IgA and IgM: } 1+\text { finely granular } \\
\text { focal capillary positivity. } \\
\text { IgG: } 3+\text { finely granular diffuse } \\
\text { capillary positivity. } \\
\text { C3: Negative. } \\
\text { C1q: } 1+\text { diffuse capillary } \\
\text { Kappa: } 2+\text { diffuse capillary } \\
\text { Lambda: } 1+\text { diffuse capillary }\end{array}$ & Diffuse & $\begin{array}{l}\text { Intramembranous } \\
\text { and subepithelial }\end{array}$ & Lupus nephritis \\
\hline 25 & 23 & $\mathrm{~F}$ & $\begin{array}{l}\operatorname{lgA} \text {, IgG, IgM and C3: } 2+\text { focal } \\
\text { capillary and mesangial } \\
\text { Kappa and Lambda: } 2+\text { focal } \\
\text { capillary and mesangial }\end{array}$ & Focal & $\begin{array}{l}\text { Subendothelial, } \\
\text { mesangial and } \\
\text { paramesangial } \\
\text { areas }\end{array}$ & Lupus nephritis \\
\hline
\end{tabular}


Table 1B: Immunoflourescence, electron microscopic findings, and pathological diagnosis in 25 patients with various glomerulopathies.

B. Non-immunocomplex-related glomerulopathies.

\begin{tabular}{|c|c|c|c|c|c|c|}
\hline & \multirow[t]{2}{*}{ Case \# } & \multirow[t]{2}{*}{ Age } & \multirow[t]{2}{*}{$\begin{array}{l}\text { Sex } \\
\text { M:male } \\
\text { F:female }\end{array}$} & $\begin{array}{l}\text { Immunoflourescence } \\
\text { Immunoglobulin and } \\
\text { complement }\end{array}$ & $\begin{array}{l}\text { Electron Microscopic } \\
\text { Findings }\end{array}$ & \multirow[t]{2}{*}{ Pathological Diagnosis } \\
\hline & & & & $\begin{array}{l}\text { Effacement of the } \\
\text { epithelial cell foot } \\
\text { processes }\end{array}$ & $\begin{array}{l}\text { Electron dense } \\
\text { immune deposits and } \\
\text { other findings }\end{array}$ & \\
\hline 2 & 39 & M & $\begin{array}{l}\text { Focal mesangial } \\
\text { deposition of } \lg \mathrm{A}(\mathrm{H}) \text { and } \\
\mathrm{C} 3(1+) \text { in the glomeruli }\end{array}$ & Focal & $\begin{array}{l}\text { Subendothelial space } \\
\text { widening by electron } \\
\text { lucent material }\end{array}$ & $\begin{array}{l}\text { Focal and segmental } \\
\text { glomerulosclerosis } \\
\text { secondary to functional } \\
\text { and structural } \\
\text { adaptations }\end{array}$ \\
\hline 6 & 41 & $\mathrm{~F}$ & $\begin{array}{l}\text { IgA, IgG, IgM: Negative. } \\
\text { C3: Trivial }(1+) \text { focal } \\
\text { mesangial positivity. } \\
\text { C3: Trivial }(1+) \text { focal } \\
\text { mesangial positivity. }\end{array}$ & $\begin{array}{l}\text { Global sclerosis of only } \\
\text { one glomerulus present }\end{array}$ & $\begin{array}{l}\text { Global sclerosis of only } \\
\text { one glomerulus present }\end{array}$ & $\begin{array}{l}\text { Focal and segmental } \\
\text { glomerulosclerosis }\end{array}$ \\
\hline 7 & 24 & M & $\begin{array}{l}\text { IgM: Mild }(2+) \text { diffuse } \\
\text { mesangial positivity }\end{array}$ & Diffuse & microvillus formation & $\begin{array}{l}\text { Advance focal } \\
\text { and segmental } \\
\text { glomerulosclerosis, }\end{array}$ \\
\hline 11 & 53 & M & $\begin{array}{l}\lg A, \lg G \text { and } \lg M: C 3: 1+ \\
\text { focal mesangial positivity. } \\
\text { C1 : Negative. }\end{array}$ & Focal & - & $\begin{array}{l}\text { Focal and segmental } \\
\text { glomerulosclerosis }\end{array}$ \\
\hline 15 & 56 & M & $\begin{array}{l}\text { IgA, IgG, IgM and C3: } \\
\text { Negative. } \\
\text { Kappa: Negative in the } \\
\text { glomeruli. Casts are } \\
\text { positive. } \\
\text { Lambda: Negative in } \\
\text { the glomeruli. Casts are } \\
\text { negative. } \\
\text { Fibrinogen: Non-specific } \\
\text { interstitial staining. }\end{array}$ & Focal & - & $\begin{array}{l}\text { Myeloma cast } \\
\text { nephropathy with } \\
\text { associated acute tubular } \\
\text { injury }\end{array}$ \\
\hline
\end{tabular}

Table 2: Level of proteinuria, average number of podocytes/glomerulus, and average number of podocytes with reabsorbed intracytoplasmic proteins/glomerulus in 25 patients with various glomerulopathies.

\begin{tabular}{|c|c|c|c|c|c|}
\hline Case & Age & $\begin{array}{l}\text { Sex } \\
\text { Male,M; } \\
\text { Female,F }\end{array}$ & $\begin{array}{l}\text { Proteinuria }(\mathrm{gm} / \text { day) } \\
\text { (Normal range }=0.01-0.15 \\
\text { gm/day) }\end{array}$ & $\begin{array}{l}\text { Average number of } \\
\text { Podocytes/glomerulus }\end{array}$ & $\begin{array}{l}\text { Average number of } \\
\text { podocytes with reabsorbed } \\
\text { proteins/glomerulus }\end{array}$ \\
\hline 1 * & 33 & $\mathrm{~F}$ & 2.32 & 26 & 3 \\
\hline $2^{* *}$ & 39 & M & 1.02 & 21 & 3 \\
\hline $3^{*}$ & 50 & $M$ & 2.27 & 23 & 3 \\
\hline $4^{*}$ control & 34 & $\mathrm{~F}$ & 0.16 & 22 & 0 \\
\hline $5^{*}$ & 17 & $\mathrm{~F}$ & 0.89 & 25 & 3 \\
\hline $6^{* *}$ & 41 & $\mathrm{~F}$ & 1.11 & 23 & 3 \\
\hline $7^{\star *}$ & 24 & $M$ & $3+$ & 24 & 2 \\
\hline $8^{*}$ & 67 & M & 13.83 & 26 & 1 \\
\hline $9^{*}$ & 25 & $\mathrm{~F}$ & 3.46 & 23 & 2 \\
\hline $10^{*}$ & 20 & $\mathrm{~F}$ & 28.97 & 20 & 1 \\
\hline $11^{* *}$ control & 53 & $M$ & 0.14 & 22 & 0 \\
\hline
\end{tabular}




\begin{tabular}{|l|l|l|l|l|l|}
\hline $12^{*}$ & 48 & M & 1.71 & 21 & 3 \\
\hline $13^{*}$ & 48 & M & 1.54 & 25 & 5 \\
\hline $14^{*}$ & 25 & F & $2+$ & 21 & 4 \\
\hline $15^{* *}$ & 56 & M & 4.24 & 20 & 2 \\
\hline $16^{*}$ & 16 & M & 6.14 & 23 & 1 \\
\hline $17^{*}$ control & 6 & M & 0.01 trace & 21 & 0 \\
\hline $18^{*}$ & 36 & F & 0.60 & 24 & 3 \\
\hline $19^{*}$ & 16 & M & 3.69 & 26 & 2 \\
\hline $20^{*}$ & 50 & F & 0.49 & 25 & 3 \\
\hline $21^{*}$ & 2 & F & $4+$ & 21 & 1 \\
\hline $22^{*}$ & 49 & F & 1.35 & 23 & 3 \\
\hline $23^{*}$ & 46 & M & 2.60 & 22 & 2 \\
\hline $24^{*}$ & 30 & F & 0.58 & 23 & 3 \\
\hline $25^{*}$ & 23 & F & 1.78 & 20 & 3 \\
\hline
\end{tabular}

"Immunocomplex deposition-related glomerulonephritis; "Non-immunocomplex-related glomerulopathies.

Table 3: Groups of cases with various numbers of glomerular podocytes with reabsorbed intracytoplasmic proteins/glomerulus; Three to five podocytes (high), Two podocytes (moderate), One podocyte (low), and the average level of proteinuria in each group.

\section{Group}

3-5 podocytes (high)

2 podocytes (moderate)

1 podocyte (low)

\section{Cases}

$1,2,3,5,6,12,13,14,18,20,22,24,25$

$7,9,15,19,23$

$8,10,16,21$
Average level of proteinuria (gm / day)

1.36

3.40

13.24

Table 4: Albumin and immunofluorescence scores of tubular reabsorbed proteins, tubular cell injury and level of proteinuria in patients with various glomerulopathies.

\begin{tabular}{|c|c|c|c|c|}
\hline Case \# & $\begin{array}{l}\text { Reabsorbed Albumin } \\
\text { Scores }^{*}\end{array}$ & $\begin{array}{l}\text { Immunoflourescence } \\
\text { scores }^{* *}\end{array}$ & Tubular Cell Injury & $\begin{array}{l}\text { Level of Proteinuria (gm/day) } \\
\text { (Normal }=0.01-0.15 \mathrm{gm} / \text { day) }\end{array}$ \\
\hline 1 & $1+$ & Mild & + & 2.32 \\
\hline 2 & $3+$ & Moderate & ++ & 1.02 \\
\hline 3 & $3+$ & Moderate & ++ & 2.27 \\
\hline 4 & 0 & Negative & 0 & 0.16 \\
\hline 5 & $3+$ & Severe & ++ & 0.89 \\
\hline 6 & $3+$ & Moderate & + & 1.11 \\
\hline 7 & 0 & Negative & 0 & $3+$ \\
\hline 8 & 0 & Negative & 0 & 13.83 \\
\hline 9 & 0 & Negative & 0 & 3.46 \\
\hline 10 & 0 & Negative & + & 28.97 \\
\hline 11 & 0 & Negative & + & 0.14 \\
\hline 12 & $3+$ & Moderate & ++ & 1.71 \\
\hline 13 & $3+$ & Moderate & ++ & 1.54 \\
\hline 14 & $3+$ & Moderate & ++ & $2+$ \\
\hline 15 & 0 & Negative & 0 & 4.24 \\
\hline 16 & 0 & Negative & + & 6.14 \\
\hline 17 & 0 & Negative & 0 & 0.01 trace \\
\hline 18 & $3+$ & Moderate & ++ & 0.60 \\
\hline 19 & 0 & Negative & 0 & 3.69 \\
\hline 20 & $3+$ & Moderate & ++ & 0.49 \\
\hline 21 & 0 & Negative & 0 & $4+$ \\
\hline 22 & $3+$ & Moderate & ++ & 1.35 \\
\hline
\end{tabular}




\begin{tabular}{|l|l|l|l|l|}
\hline 23 & $3+$ & Moderate & ++ & 2.60 \\
\hline 24 & $3+$ & Moderate & ++ & 0.58 \\
\hline 25 & $3+$ & Moderate & ++ & 1.78 \\
\hline
\end{tabular}

"Reabsorbed albumin scores as observed by light microscopy in renal tubular cells:

0 : No significant hyaline reabsorption droplet change in tubular cells cytoplasm; $1+:$ Minimal hyaline reabsorption droplet change in tubular cells cytoplasm; 2+: Mild hyaline reabsorption droplet change in tubular cells cytoplasm; $3+$ : Moderate hyaline reabsorption droplet change in tubular cells cytoplasm

${ }^{*}$ Immunofluorescence scores:

Negative, no reabsorption proteins; Mild, less than $25 \%$ of the tubules show reabsorption proteins; Moderate, $26 \%$ to $50 \%$ of the tubules show reabsorption proteins; Severe, more than $50 \%$ of the tubules show reabsorption proteins.

***Tubular cell injury:

0 : No significant tubular cell changes; +Mild tubular cell injury in the form of cytoplasmic vacuolar change; ++Moderate tubular cell injury in the form of cytoplasmic vacuolation, apical blebbings, partial loss of brush border.

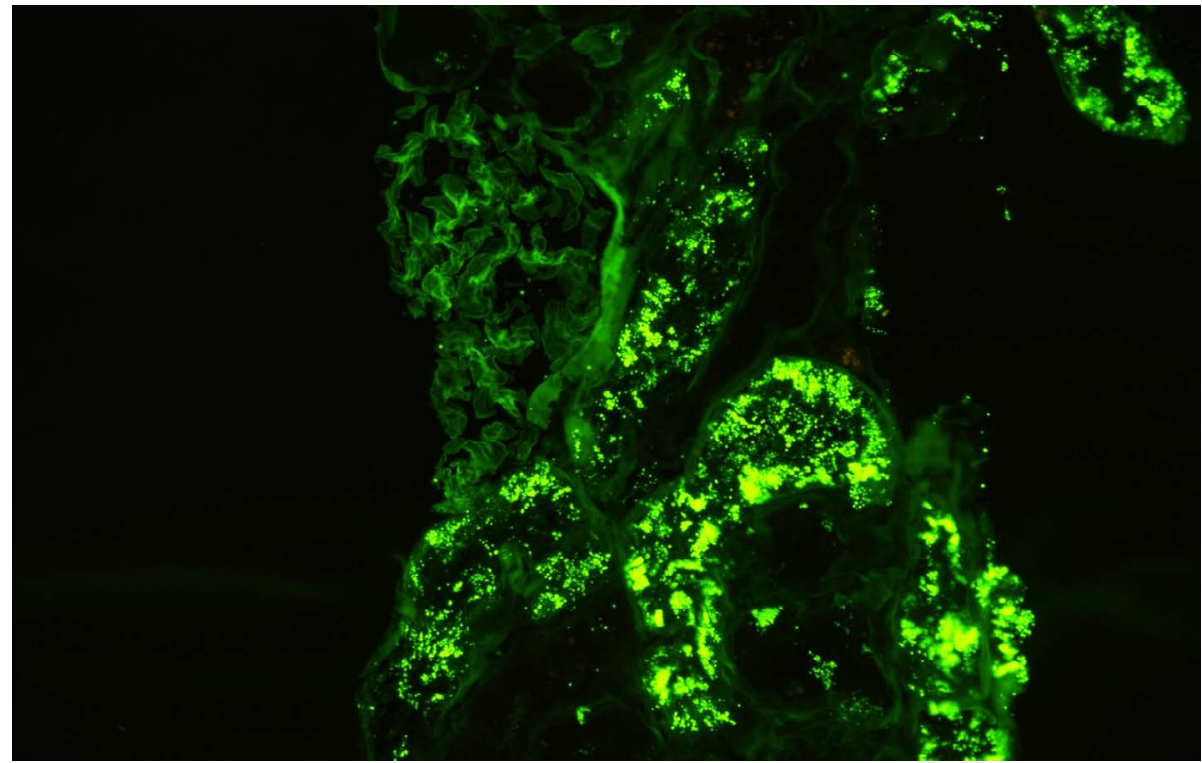

Figure 1: Immunofluorescence micrograph of a renal tissue showing severe IF staining, more than $50 \%$ of the tubules show positivity for reabsorbed albumin in their lining epithelium.

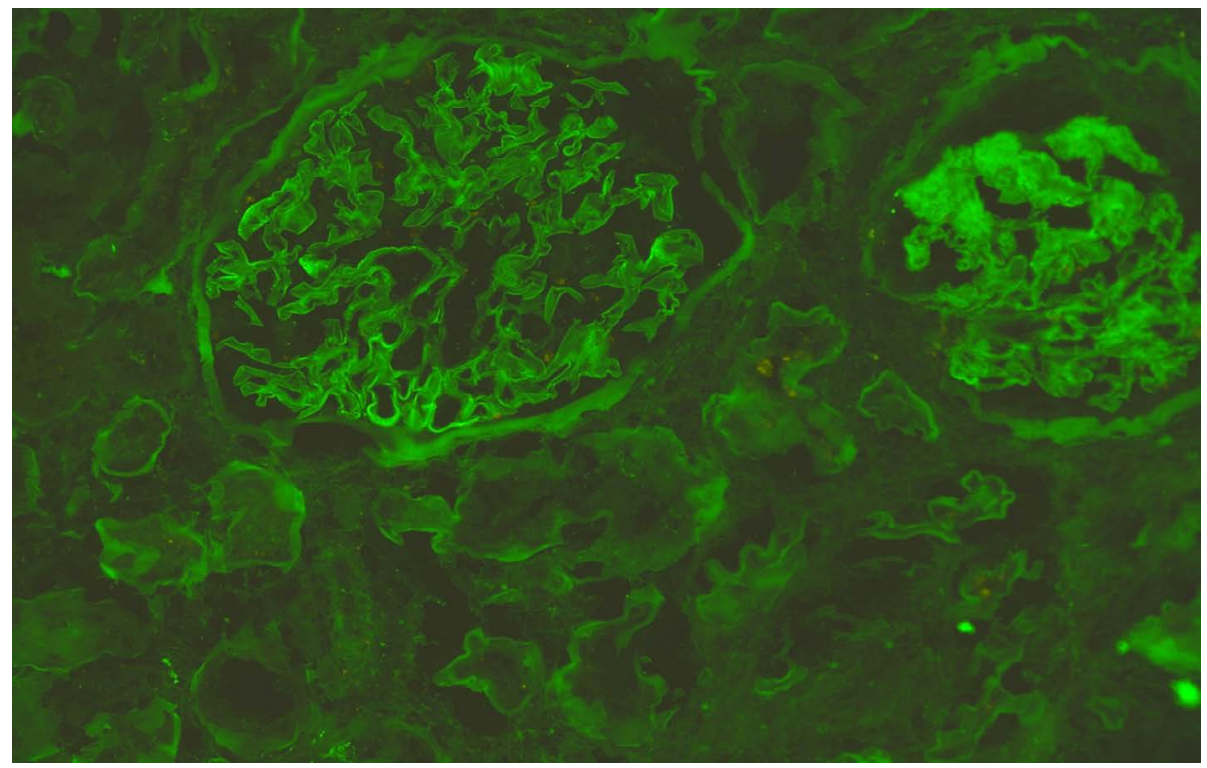

Figure 2: Immunofluorescence micrograph of a renal tissue show negative tubular IF staining for reabsorbed albumin. 
Figure 1 and Figure 2 show the immunofluorescence (IF) staining in two different cases; a case with mild $I F$, less than $25 \%$ of the tubules showing reabsorption proteins (Figure 1), and another case with severe IF, more than $50 \%$ of the tubules show reabsorption proteins (Figure 2).
Transmission electron microscopy revealed the presence of glomerular podocytes with low and highreabsorbed intracytoplasmic protein droplets in the examined cases (Figure 3 and Figure 4 respectively). The reabsorbed proteins were recognized as highly electron dense amorphous structures. Prevalence of the reabsorbed proteins in podocytes varied among

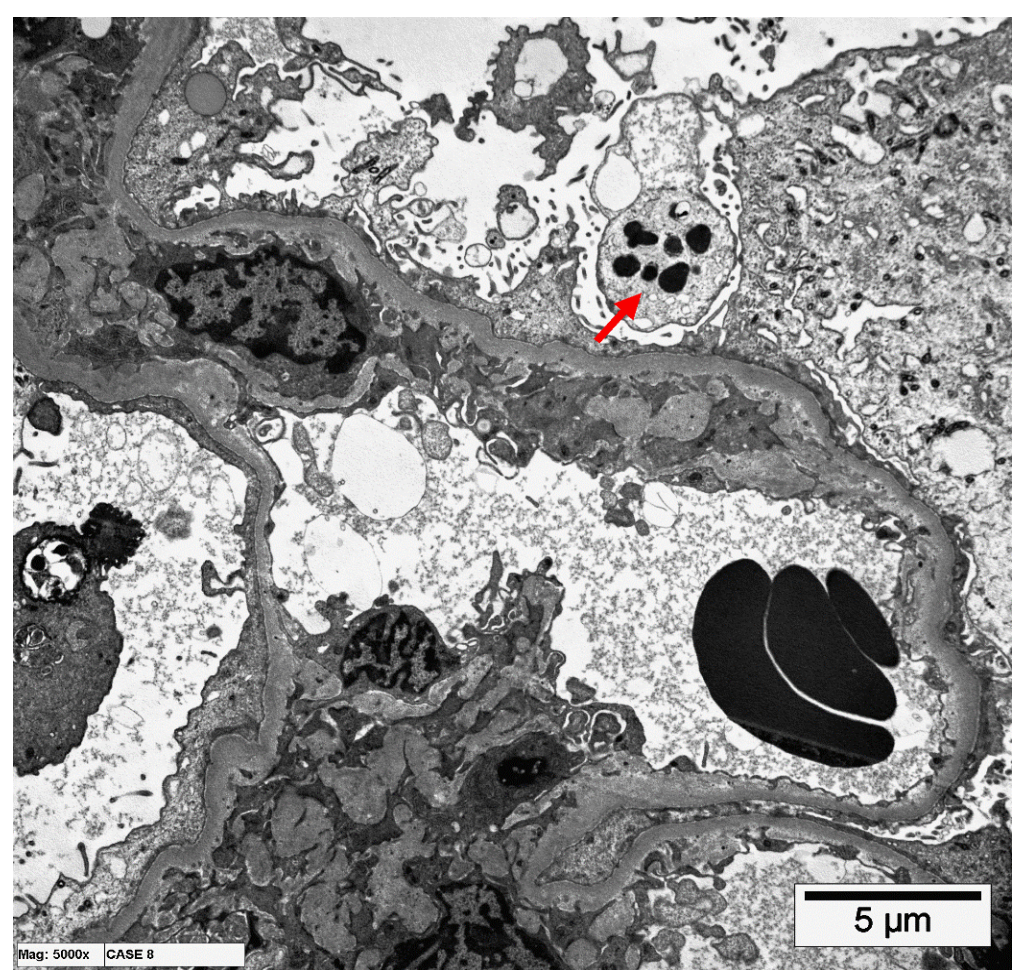

Figure 3: Transmission electron micrographs showing glomerular podocytes with low reabsorbed intracytoplasmic proteins (arrows). (uranyl acetate, lead citrate, x6000).

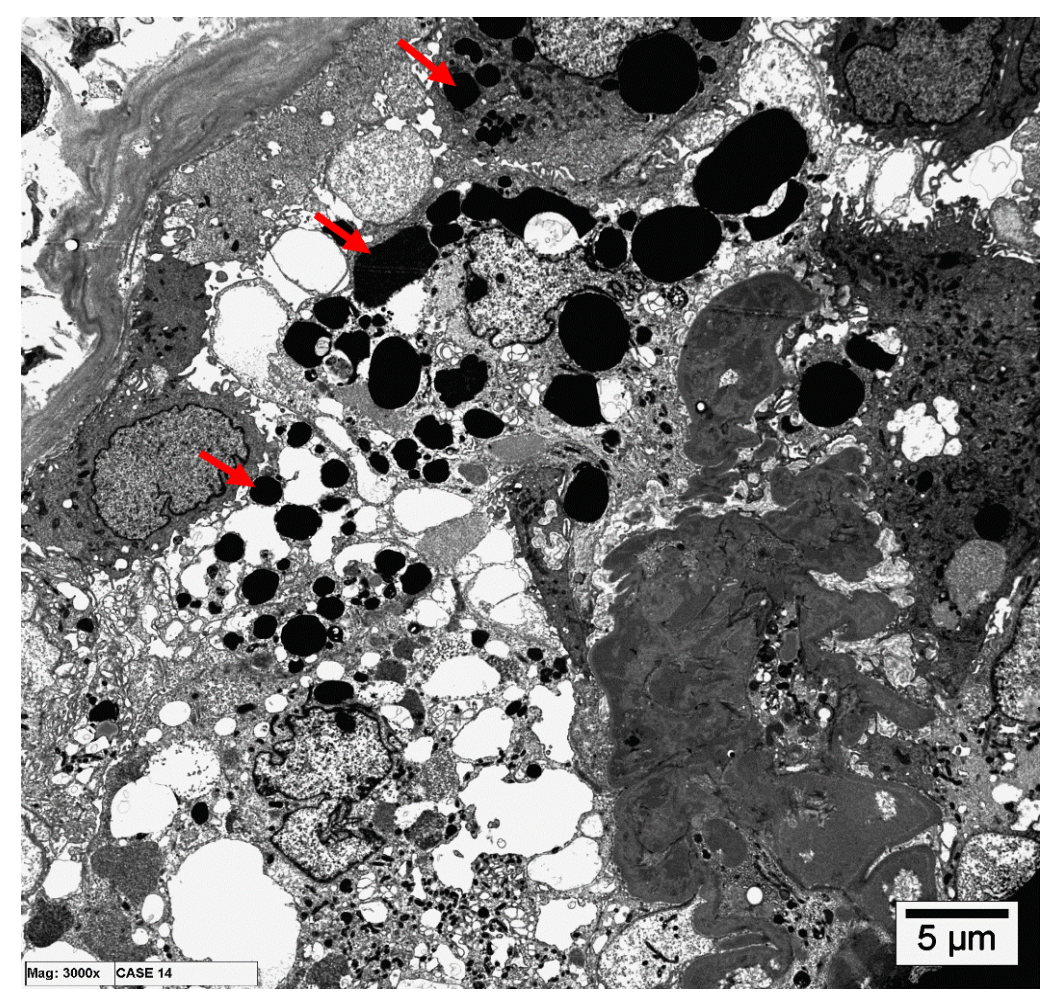

Figure 4: Transmission electron micrographs showing glomerular podocytes with high reabsorbed intracytoplasmic proteins (arrows). (uranyl acetate, lead citrate, x6000). 


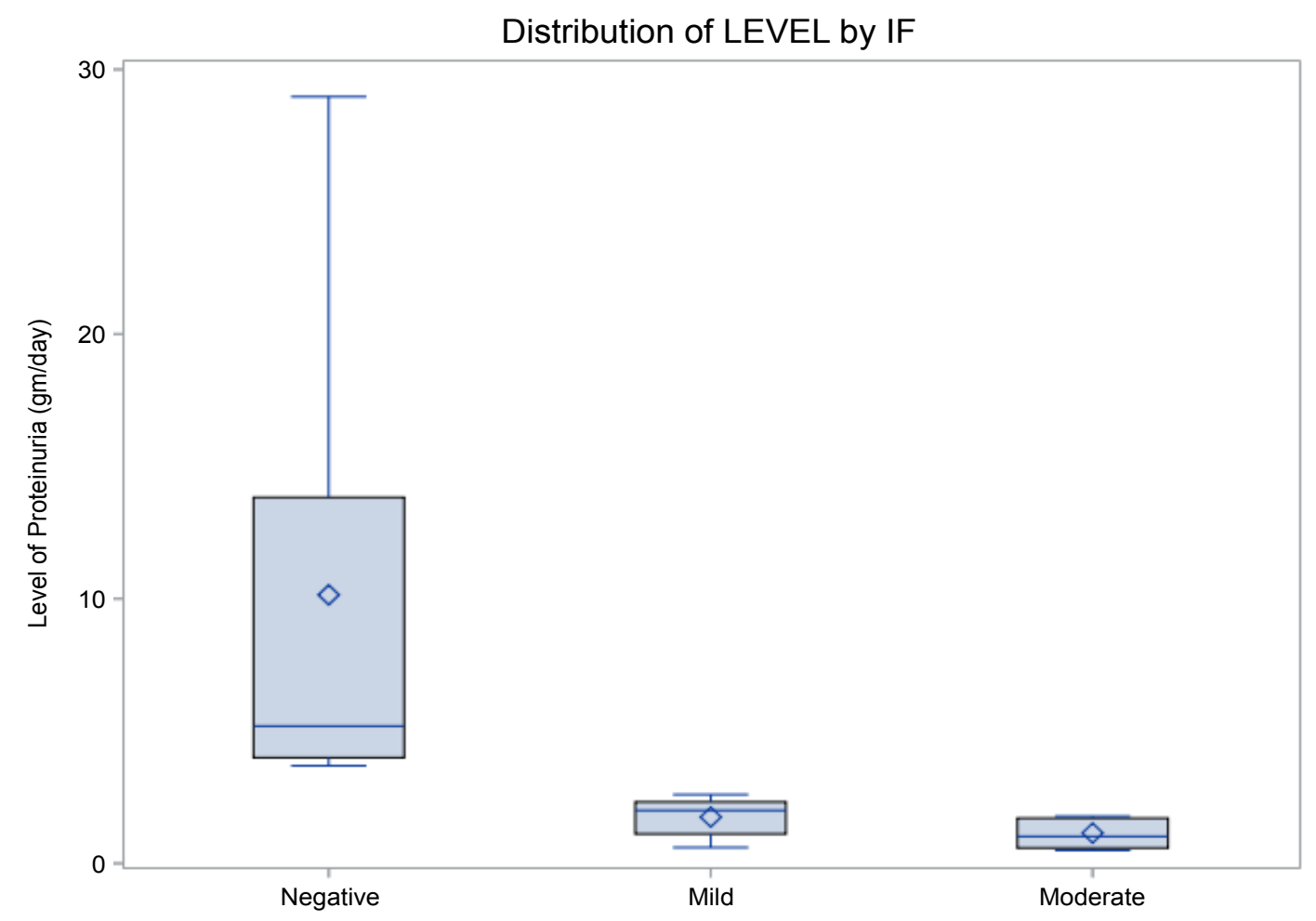

Figure 5: Statistical boxplot showing negative correlation between IF score and various levels of proteinuria.

Table 5: Statistical analysis of data relevant to the count of podocytes with reabsorbed intracytoplasmic proteins in the different groups of cases in relation to the level of proteinuria in each group.

\begin{tabular}{|c|c|c|c|c|c|c|c|c|}
\hline \multirow[t]{2}{*}{ Group } & \multirow[t]{2}{*}{$\mathbf{N}$} & \multirow{2}{*}{$\begin{array}{l}\text { Mean of } \\
\text { proteinuria level }\end{array}$} & \multirow{2}{*}{$\begin{array}{l}\text { Std. } \\
\text { Deviation }\end{array}$} & \multirow{2}{*}{ Std. Error } & \multicolumn{2}{|c|}{ 95\% Confidence Interval for Mean } & \multirow[t]{2}{*}{ Minimum } & \multirow[t]{2}{*}{ Maximum } \\
\hline & & & & & Lower Bound & Upper Bound & & \\
\hline Low-Moderate* & 9 & $5.8533^{* *}$ & 8.16011 & 2.35562 & 0.6686 & 11.0380 & 0.01 & 28.97 \\
\hline High $^{*}$ & 13 & $1.3585^{* *}$ & 0.63460 & 0.17601 & 0.9750 & 1.7419 & 0.49 & 2.32 \\
\hline Total & 22 & 3.5160 & 5.99780 & 1.19956 & 1.0402 & 5.9918 & 0.01 & 28.97 \\
\hline
\end{tabular}

"Low-moderate and high groups of cases with one-two, three-five podocytes with reabsorbed proteins, respectively; "*Significant difference at $p$-value $<0.05$.

the different cases and also varied from area to another within the same glomerulus. Podocytes containing cytoplasmic reabsorbed protein droplets were enlarged and disclosed disrupted cytoplasmic organelles and deteriorated surface microvilli.

Table 5 summarizes the statistical analysis of the obtained glomerular data. Low and moderate groups indicated in Table 3 were merged in one group to be statistically analyzed against the high group. The analysis approved significant differences between the studied groups. No significant differences were detected among cases of different sex and age.

Statistical analysis of the renal tubular data is shown in a boxplot (Figure 5). A significant negative correlation between the Immunofluorescence tubular scores and level of proteinuria was detected by the statistical analysis.

\section{Discussion}

Presently, the correlation between the morphologi- cally abnormal podocytes and renal tubular epithelium containing reabsorbed intracytoplasmic proteins and the level of proteinuria was evaluated.

Normal urinary protein excretion is < $150 \mathrm{mg} / 24$ hour, and daily albumin excretion in a normal person is $<30 \mathrm{mg}$. 11 Loss of proteins in urine is the hallmark of tubular and glomerular diseases, and may arise from structural and/or functional alterations involving different cell types [19-21]. Various proteinuric diseases may share similar renal pathological changes and may have a common progression of renal injury [6,22-25]. Albuminuria is strongly associated with the progression of renal disease [26-28].

Proteinuria of the presently studied cases was most likely of glomerular origin, i.e., at the glomerular level, based upon the encountered glomerular structural changes. Beside the well-known protein endocytosis at the renal proximal tubular cells, there is an increasing evidence of glomerular protein handling by podocytes [22,29-31]. It has been concluded that identifying the 
mechanisms involved in albumin handling in podocytes is essential to understand the pathogenesis of various glomerulopathies [19]. Such mechanisms in human podocytes are committed to internalizing albumin (albumin endocytosis) through a receptor-mediated mechanism [29].

The structural integrity of podocytes is crucial to guard against leakage of proteins in urine. In this regard, podocytopathies are the most common group of glomerular disorders leading to proteinuria $[1,19]$. The final clinical scoring of proteinuria is largely related to the podocytes integrity and activity in reabsorbing leaked proteins.

The present results clearly indicate that cases of glomerulopathies with the higher average number of podocytes containing reabsorbed cytoplasmic protein droplets had the lower levels of proteinuria. Subsequently, it can be concluded that the larger the number of morphologically recognizable podocytes containing reabsorbed proteins, the lower the level of the expected clinical proteinuria.

Hyper-filtration of proteins is known to be followed by increased reabsorption in the renal proximal tubules [12]. The basic defects leading to tubular proteinuria arise from proximal tubules, with the result of excretion of proteins that are normally reabsorbed efficiently by the proximal tubular cells through a receptor-mediated endocytosis [6].

The reabsorbed proteins can be cytotoxic to proximal tubular epithelium and extensive reabsorption of large quantity of abnormally filtered proteins may provoke tubular damage $[17,24]$.

Similar to glomerular Podocytes, active reabsorption of filtered proteins by proximal tubular cells is done by endocytosis by the crucial aid of endocytic surface receptors, such as megalin and cubilin as a form of receptor-mediated process $[1,5,12]$. Changes in the expression and/or subcellular distribution of these two endocytic receptors are expected to be associated with proteinuria due to receptor dysfunction [32,33].

The present data, regardless the type of the underlying glomerulopathies, showed that the greatest average number of podocytes with distinct reabsorbed proteins and the highest immunofluorescence tubular score were detected in cases with lower proteinuria levels. In contrast, cases with the highest proteinuria level had the fewer number of podocytes containing reabsorbed proteins and the lowest IF tubular score. Subsequently, the level of proteinuria and the progression of a proteinuric disease can be predicted through estimation of these parameters.

These findings might indicate a parallel and synergic glomerular and tubular roles to handle, retain and preserve proteins and thus hindering protein leakage into urine and reabsorb proteins that may leak into the glomerular filtrate.

The present work was concentrated objectively on the correlation between the morphologically recognizable reabsorbed proteins in glomerular podocytes and renal tubular epithelium, and the level of clinical proteinuria in patients with glomerulopathies. The current study may hopefully constitute a base for the next investigations concerned with the structural changes of glomerular podocytes and renal tubular epithelium as a compensative mechanism in cases of proteinuria.

However, further investigations on a larger scale of patients with clinical proteinuria are needed to strongly establish the currently evaluated correlation.

\section{Acknowledgment}

The author would like to thank Dr. Mohammed Mubarak for his efforts in the Electron Microscopy technical support (King Saud University) and Dr. Albara Marwa for statistical analysis (King Saud University).

\section{Declaration of Interest with Statement}

I have no relevant interests to disclose. This research is not funded by any agency.

\section{References}

1. Birn H, Fyfe JC, Jacobson C, Mounier F, Verroust PJ, et al. (2000) Cubulin is an albumin binding protein important for renal tubular reabsorption. J Clin Inves 105: 1353-1361.

2. Brinkkoetter PT, Ising C, Benzing T (2013) The role of the podocyte in albumin filtration. Nat Rev Nephrol 9: 328-336.

3. Christensen El, Bjerke T (1986) Renal tubular uptake of protein: Effect of pH. Renal Physiol 9: 160-166.

4. Maha A (2018) Correlation between reabsorbed intracytoplasmic proteins in glomerular podocytes and the level of proteinuria in patients with glomerulopathies. Ultrastruct Pathol 42: 489-497.

5. Christensen El, Birn H (2002) Megalin and cubilin: Multifunctional endocytic receptors. Nat Rev Mol Cell Biol 3: $256-266$

6. Christensen El, Glurek J (2004) Protein reabsorption in renal proximal tubule- function and dysfunction in kidney pathophysiology. Pediatric Nephrology 19: 714-721.

7. Christensen EI, Rennke HG, Carone FA (1983) Renal uptake of protein: effect of molecular charge. Am J Physiol 244: F436-F441.

8. Han SS, Ahn SY, Ryu J, Baek SH, Chin HJ, et al. (2014) Proteinuria and hematuria are associated with acute kidney injury and mortality in critically ill patients. A retrospective observational study. BMC Nephrol 15: 39.

9. Jonsson AL, Grangvist A, Elvin J, Johansson ME, Haraldsson B, et al. (2014) Effects of melanocortin 1 receptor agonists in experimental nephropathies. PLoS one 9: e87816.

10. Piskinpasa $\mathrm{S}$, Altun $\mathrm{B}$, Akoglu $\mathrm{H}$, Yildirim $\mathrm{T}$, Agbaht $\mathrm{K}$, et al. (2013) An uninvestigated risk factor for contrast-induced nephropathy in chronic kidney disease: Proteinuria. Ren Fail 35: 62-65. 
11. Rosenberg ME, Hostetler C (2003) Proteinuria. In: Seldin DW, Giebisch G, ( $2^{\text {nd }}$ edn), The kidney: Physiology and pathophysiology. Raven, New York, 3039-3061.

12. Christensen El, Birn H, Verroust P, Moestrup SK (1998) Membrane receptors for endocytosis in the renal proximal tubule. Int Rev Cytol 180: 237-284.

13. Christensen El, Moskaug JO, Vorum H, Jacobsen C Gundersen TE, et al. (1999) Evidence for an essential role of megalin in transepithelial transport of retinol. Am J soc nephrol 10: 685-695.

14. Dai H, Liu Q, Liu B (2017) Research progress on mechanism of podocyte depletion in diabetic nephropathy. J Diabetes Res 2017.

15. Schmieder RE, Mann JF, Schumacher H, Gao P, Mancia G, et al. (2011) Changes in albuminuria predict mortality and morbidity in patients with vascular disease. J Am Soc Nephrol 22: 1353-1364.

16. Yang JQ, Ran P, Chen JY, He YT, Li LW, et al. (2014) Development of contrast-induced acute kidney injury after elective contrast media exposure in patients with type 2 diabetes mellitus: Effect of albuminuria. PLos ONE 9: e106454.

17. De Souza DB, Gregorio BM, Benchimol M, de Morais Nascimento FA (2016) Evaluation of the glomerular filtration barrier by electron microscopy. Open Acccess Books, INTECH.

18. Hayashi D, Yagi, K, Song C, Ueda S, Yamanoue M, et al (2017) Diacylglycerol kinase alpha is involved in the vitamin E-induced amelioration of diabetic nephropathy in mice. Sci Rep 7: 2597.

19. Dobrinskikh E, Okamura K, Kopp JB, Doctor RB, Blaine J (2014) Human podocytes perform polarized, caveolaedependent albumin endocytosis. Am J Physiol Renal Pysiol 306: 941-951.

20. Krtil J, Platenik J, Kazderova M, Tesar V, Zima T (2007) Culture methods of glomerular podocytes. Kidney Blood Press Res 30: 162-174.

21. Lerma EV (2017) Proteinuria. Medscape.

22. Gianesello L, Priante G, Ceol M, Radu CM, Saleem MA, et al. (2017) Albumin uptake in human podocyte: A possible role for the cubulin-amnionless (CUBAM) complex. Scientific Reports 7: 13705
23. Jafar TH, Stark PC, Schmid CH, Landa M, Maschio G, et al. (2001) Proteinuria as a modifiable risk factor for the progression of non-diabetic renal disease. Kidney Int 60 : 1131-1140.

24. James MT, Hemmelgarn BR, Wiebe N, Pannu N, Manns $\mathrm{BJ}$, et al. (2010) Glomerular filtration rate, proteinuria, and the incidence and consequences of acute kidney injury: A cohort study. Lancet 376: 2096-2103.

25. Michael Gekle (1998) Renal proximal tubular epithelium reabsorption: Daily prevention of albuminuria. News Physiol Sci 13: 5-11.

26. Eyre J, loannou K, Grubb BD, Saleem MA, Mathieson PW, et al. (2007) Statin-sensitive endocytosis of albumin by glomerular podocytes. Am J Physiol 292: F674-681.

27. Gansevoort RT, Navis GJ, Wapstra FH, de Jong PE, de Zeeuw D (1997) Proteinuria and progression of renal disease: Therapeutic implications. Curr Opin Nephro Hypertens 6: 133-140.

28. Gerstein HC, Mann JF, Yi Q, Zinman B, Dinneen SF, et al. (2001) Albuminuria and risk of cardiovascular events, death, and heart failure in diabetic and nondiabetic individuals. JAMA 286: 421-426.

29. Gianesello L, Ceol M, Priante G, Anglani F, Prete D (2016) Proteins lost and found: not only a tubular matter. J Clinical Nephrol Kidney Dis 1: 1002.

30. Grams ME, Astor BC, Bash LD, Matsushita K, Wang Y, et al. (2010) Albuminuria and estimated glomerular filtration rate independently associate with acute kidney injury. J AM Soc Nephrol 21: 1757-1764.

31. Grant Otto (2017) Podocyte biology: Proteolytic processing in podocytes. Nature Rev Nephrol 13: 596.

32. Kinugasa S, Tojo A, Sakai T, Tsumura H, Takahashi M, et al. (2011) Selective albuminuria via podocyte albumin transport in puromycin nephrotic rats is attenuated by an inhibitor of NADPH oxidase. Kidney Int 80: 1328-1338.

33. Wehlstedt-Froberg V, Petterson, T, Aminoff $M$, Dugué $B$, Gräsbeck R (2003) Proteinuria in cubulin-deficient patients with selective vitamin B12 malabsorption. Pediatr Nephrol 18: $417-421$ 